\title{
Downward current electron beams observed at the dipolarization front
}

\author{
ZHENG Hao, FU SuiYan*, ZONG QuiGang \& PU ZuYin \\ School of Earth and Space Sciences, Peking University, Beijing 100871, China
}

Received July 3, 2012; accepted August 22, 2012; published online October 20, 2012

The strong field-aligned pitch angle distribution of electrons is observed right at the dipolarization front (DF) before the arriving of a high speed flow when the four Cluster satellites are traveling in the magnetotail around $15 \mathrm{R}_{\mathrm{E}}$ on July 22, 2001. The increased electron fluxes only last for a short time period at the DF, corresponding to just a few bouncing periods for $1 \mathrm{keV}$ electrons. The field-aligned current contributed by these electrons agrees well with that calculated by the magnetic field observations by four satellites at the front. These electron streams are found in the energy range of $0.2-2 \mathrm{keV}$, peak around $1 \mathrm{keV}$. It is suggested that these downward current electrons may be originated near the aurora region by some kinds of potential structure. The occurrence of these electrons implies that the formation of the dipolarization front and the associated field-aligned current play an important role in the magnetosphere-ionosphere coupling.

plasma sheet, high speed flow, electron beam, dipolarization front, field-aligned current

Citation: Zheng H, Fu S Y, Zong Q G, et al. Downward current electron beams observed at the dipolarization front. Chin Sci Bull, 2013, 58: 913-919, doi: $10.1007 / \mathrm{s} 11434-012-5478-3$

Dipolarization is a significant dynamic process in the magnetotail, in which the magnetic field topology changes from tail-like to more dipole-like in a relative short time scale. Dipolarization processes are commonly observed at the time period of substorm, plasma sheet expansion or Bursty Bulk Flows (BBFs) [1-4].

The concept of dipolarization front (DF) was first proposed by Nakamura et al. [1] when they studies high speed flows. Based on the analysis of the four Cluster satellites data, it is found that, on July 22, 2001, a dipolarization front moves earthward and dawnward ahead of the high-speed earthward flow. The plasma before this front is deflected, consistent with the plasma ahead of a localized plasma bubble centered at midnight side being pushed aside by the moving obstacle. It is believed that the evolution of the dipolarization front across the tail is directly coupled with the fast flow. Multi-point observations by five THEMIS probes situated in the near-equatorial plasma sheet at distances of $\left(-20 R_{E},-10 R_{E}\right)$ are used to carefully study the dipolariza-

*Corresponding author (email: suiyanfu@pku.edu.cn) tion front event on February 27, 2009 [5]. The variations in magnetic field and plasma moments at the dipolarization front reveal characteristic signatures of BBFs: increase in bulk velocity and magnetic pressure, and decrease in plasma density and pressure. And the quick southward variation of magnetic field observed ahead of the DF has been proved to be a spatial structure associated with the propagating of DF.

Based on the observation of THEMIS on 23 February, 2008, Sergeev et al. [6] first investigated the kinetic structure of the sharp dipolarization front, which was found to be a very thin current sheet along the North-South direction embedded within an earthward-propagating flow burst. Clear finite proton gyro-radius effects are observed in the center of the thin current sheet and strong E-field bursts of the lower-hybrid time scale at the injection front with a density depletion. Since enhancement of electron fluxes are commonly observed in dipolarization events, many works focus on the energization mechanism of electrons. The energetic electron flux enhancements at the dipolarization fronts are found to be associated with large wave fluctuations extending from below the lower hybrid frequency to 
above the electron cyclotron frequency [7]. Fu et al. [8] have proposed that Fermi acceleration dominates inside a decaying flux pileup region (FPR), while betatron acceleration dominates inside an increasing FPR. It is still a debate whether the injection of electrons are associated with wave-particle interaction or simply produced by adiabatic processes.

In order to know more about the formation of the dipolarization front and its relation with electron dynamics, we revisit the event which has been studied by Nakamura et al. [1]. We, in this paper, focus on the particle behavior right at the sharp dipolarization front and try to link it with the global picture of the high speed flow and field aligned current. This paper presents observations of bidirectional electrons around $1 \mathrm{keV}$ at the dipolarization front, providing the necessary field aligned current for the forming of the thin current sheet at the DF. Data from CIS, EFW, FGM, PEACE onboard Cluster used in this study can be found in [9]. Geomagnetic solar magnetosphere (GSM) coordinates are used throughout the paper unless noted otherwise.

\section{Observations}

On July 22, 2001, the four Cluster spacecrafts all observed a dipolarization front (DF), in which sharp $B z$ increases before the arriving of a high speed flow [1], when they were traveling in the plasma sheet around $14.6 \mathrm{R}_{\mathrm{E}}$. Figure 1 shows the detailed observations of the event by Cluster 1 (C1). Figure 1(a) and (b) displays the configuration of four Cluster satellites in GSM $X-Y$ and $X-Z$ planes, with satellites located at post-midnight of the Northern Hemisphere in the plasma sheet. C1 is slightly closer to the Earth than the other three satellites, which are not far away from each other. Figure 1(c) provides the 4s-resolution magnetic data obtained from FGM for the time period of interest. C1 was located in the quiet plasma sheet and measured a steady magnetic field before $B z$ (black line) shown a sharp increase after a very short dip, which was supposed to due to the plasma compression [5]. $B z$ jumped about $12 \mathrm{nT}$ (from 3 to $15 \mathrm{nT}$ ) from $11: 34: 52$ to $11: 35: 08 \mathrm{UT}$, while $B x$ (green line) almost shown no change and $B y$ (red line) decreased from 15 to $\sim 3 \mathrm{nT}$ during the same time period. After the dipolarization, three components of magnetic field all decreased gradually, referring to a plasma sheet expansion effect or the satellite getting closer to the center plasma sheet. The dipolarization process was clearly shown in the elevation angle of the filed in Figure 1(d). Figure 1(e) shows three components of bulk flow velocity of $\mathrm{C} 1 \mathrm{ob}-$ tained from CIS-HIA instrument. $V x$ began to increase after
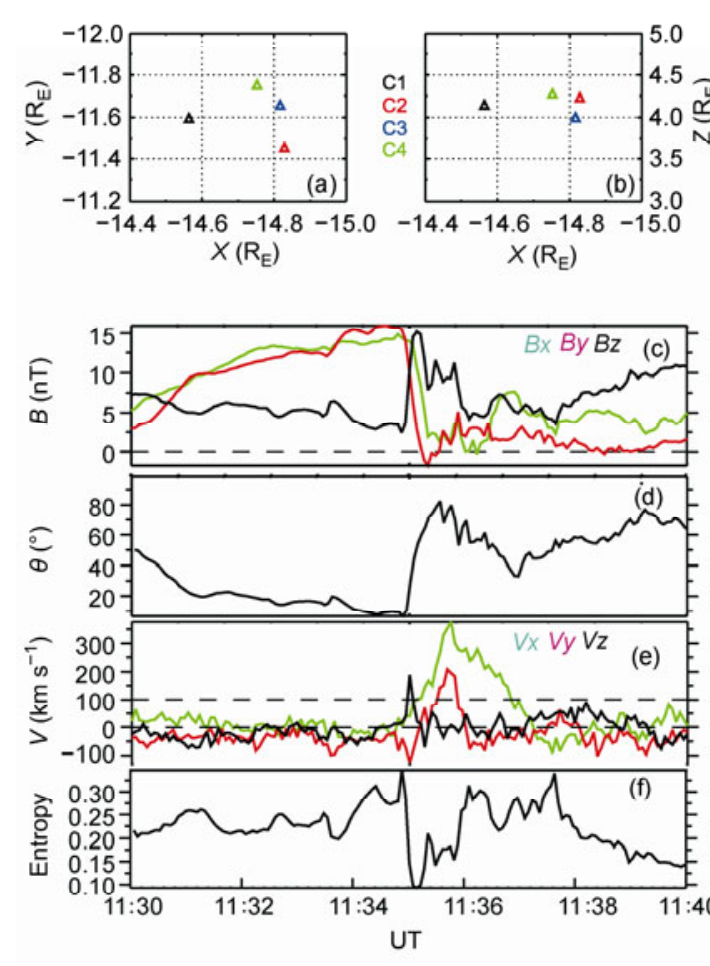

Figure 1 Overview of the observations from Cluster C1 on July 22, 2001. (a) and (b) Cluster locations in $X Y$ and $X Z$ plane; (c) magnetic field components; (d) elevation angle of the magnetic field; (e) ion flow velocity obtained from HIA; (f) entropy; (g) magnetic field magnitude (blue line) and $B z$ component (black line); (h) electron differential energy flux as a function of energy and time from PEACE and (i)-(k) electron differential energy flux of pitch angle $0^{\circ}$, $90^{\circ}, 180^{\circ}$, respectively and (1) differential energy flux of electrons (1-10 keV) as a function of pitch angle for an expanded time period. 
11:34:30 UT, reached a maximum of $400 \mathrm{~km} \mathrm{~s}^{-1}$ at about $11: 35: 45 \mathrm{UT}$, and lasted for about $2 \mathrm{~min} \mathrm{~s}^{-1}$ with a speed greater than $100 \mathrm{~km} \mathrm{~s}^{-1}$. In this high speed flow event, $V y$ changed from -100 to $250 \mathrm{~km} \mathrm{~s}^{-1}$, and $V z$ had no obvious change indicating it was moving earthward and dawn ward. The peak of flow arrived about $37 \mathrm{~s}$ after $\mathrm{Bz}$ began to increase. This kind of earthward high speed flow in the plasma sheet is defined as BBF [10] or fast flow [11] and is thought to be generated by reconnection in the tail [12]. It is also consistent with the typical "bubble" model for high speed flows [13], such as low entropy derived by $\mathrm{PV}^{-5 / 3}$ (Figure 1(f)). Figure 1(g) displays magnetic magnitude and $B z$ component for reference. The total magnetic field magnitude does not change too much (blue line) during the depolarization front, indicating a simple rotating effect of the field direction within the front. Figure 1(h) shows the electron differential energy flux obtained from PEACE instrument. An obvious increase of electron fluxes can be seen in the $0.5-10 \mathrm{keV}$ energy range, and the fluxes are at a high level after the DF all through the high speed flow period from 11:35 to 11:38. Figure 1(i)-(k) shows the electron differential energy flux for pitch angle (PA) equals to $0^{\circ}$, $90^{\circ}$ and $180^{\circ}$, respectively. It is quite clear that $0.5-2 \mathrm{keV}$ electron flux increased in $0^{\circ}$ (Figure 1(i)) and $180^{\circ}$ (Figure $1(\mathrm{k})$ ) at the DF, with slightly decrease right after the DF, and increase after the flow speed greater than $200 \mathrm{~km} \mathrm{~s}^{-1}$. On the contrary, no obvious increase for pitch angle $90^{\circ}$ electron flux (Figure 1(j)) at the DF is observed. After the $\mathrm{DF}$ an increase in the 3-10 keV energy range are shown. A pitch angle distribution of electrons at $1-10 \mathrm{keV}$ for an expanded time period is displayed at the bottom (Figure 1(l)). Obvious bi-direction anisotropy electrons are found in 1-10 $\mathrm{keV}$ (last for about 8-12 s) just during the DF time period. However, it is worth noting that $180^{\circ}$ electrons (anti-parallel to the magnetic field) have a higher flux than that of $0^{\circ}$ (parallel to the magnetic field) within the DF. Since parallel and anti-parallel electrons in this energy range are rarely distinguished within the DF structure, this phenomenon has never been reported before. And this kind of asymmetry increase of parallel and anti-parallel electron flux along with a sharp increase of $B z$ can be observed in all the other three Cluster satellites.

In order to compare the electron variation at different pitch angle quantitatively, detailed electron energy spectra before and at the DF are shown in Figure 2. The average electron differential flux from 11:34:04 to $11: 34: 28$ is shown in black diamonds, acting as background spectra before the arriving of the DF, and the electron differential flux at 11:34:56 is used to show the distribution at the DF (in red). Figure 2(a)-(c) represents electrons at $\mathrm{PA}=0^{\circ}, 90^{\circ}$, $180^{\circ}$, respectively. The spectrum of $90^{\circ}$ almost doesn't change (Figure 2(b)), while a clear increase of parallel and anti-parallel electrons is observed above $200 \mathrm{eV}$ (Figure 2(a) and (c)). We can divide the whole energy range into two parts, $0.2-2 \mathrm{keV}$ and $2-30 \mathrm{keV}$. For the higher energy part,
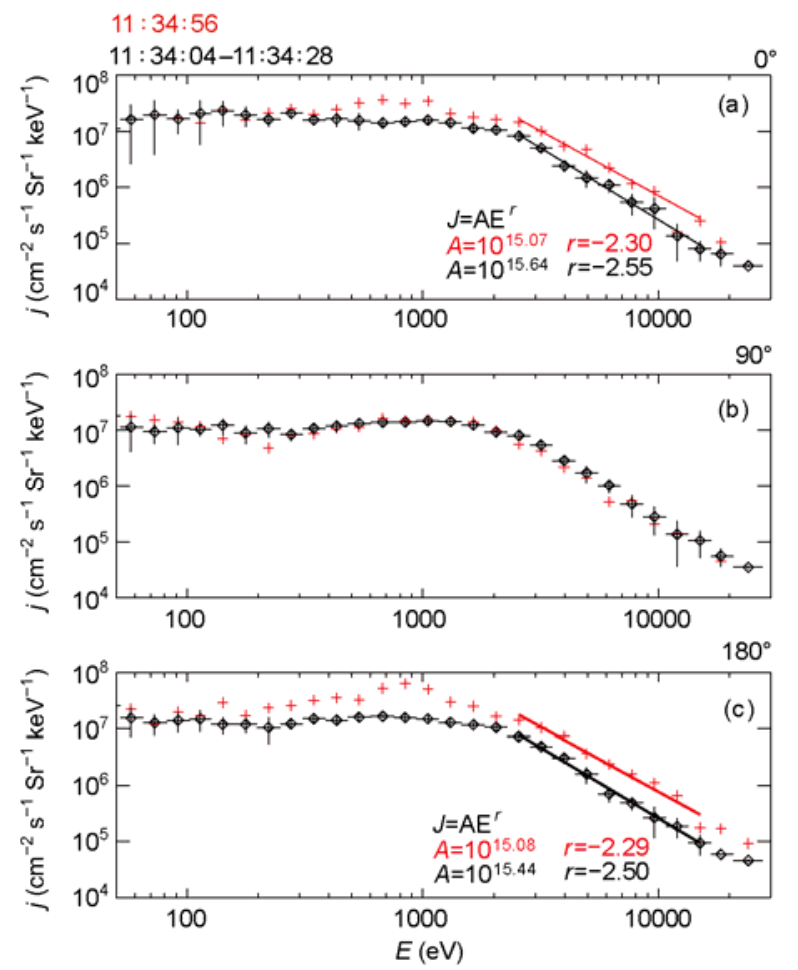

Figure 2 Energy spectra of electrons from PEACE at $\mathrm{C} 1$ for the event of 22 July 2001. (a)-(c) PA $=0^{\circ}, 90^{\circ}, 180^{\circ}$, respectively. Black diamonds are the average data from 11:34:04 to $11: 34: 28$ (before the DF), and red crosses are the data obtained at 11:34:56 (at the DF). The black and red lines are for the best fit, respectively.

we plotted the best fit and shown on the plots with black and red lines, respectively. It is interesting to note that although the electron fluxes increases for both $0^{\circ}$ and $180^{\circ}$ angle electrons, the slope of the spectra does not change much. For the smaller energy range part, anti-parallel electrons have higher differential fluxes than those of parallel electrons, indicating an asymmetry increase. The fieldaligned current carried by the asymmetry increase of parallel and anti-parallel electrons can be estimated to be 5.9 $\mathrm{nA} \mathrm{m}{ }^{-2}$.

Figure 3(a) shows the $B z$ variations of four Cluster spacecraft during the time period of the arriving of the high speed flow. The four Cluster spacecraft detected similar $B z$ increase at the dipolarization front one by one, first $\mathrm{C} 2$, then $\mathrm{C} 3$ and $\mathrm{C} 4$, and finally $\mathrm{C} 1$. As the similar magnetic field behavior has been observed in four spacecraft, timing analysis can be employed to calculate the propagation direction and velocity of this structure. The time when $B z$ reached the minimum value is set as the reference in the timing analysis, and the corresponding propagation speed is about 41.03 $\mathrm{km} \mathrm{s}^{-1}\left(V x=20.85 \mathrm{~km} \mathrm{~s}^{-1}, V y=-31.21 \mathrm{~km} \mathrm{~s}^{-1}, V z=-16.56\right.$ $\mathrm{km} \mathrm{s}^{-1}$ ). The $X$ and $Z$ component of the front is almost the same as the $X$ and $Z$ component of the plasma speed when DF is observed, while the $Y$ component of $\sim 31 \mathrm{~km} \mathrm{~s}^{-1}$ is much smaller than $V y$ of the flow $\left(\sim 110 \mathrm{~km} \mathrm{~s}^{-1}\right)$. Minimum variation analysis (MVA) method is also used to obtain the 


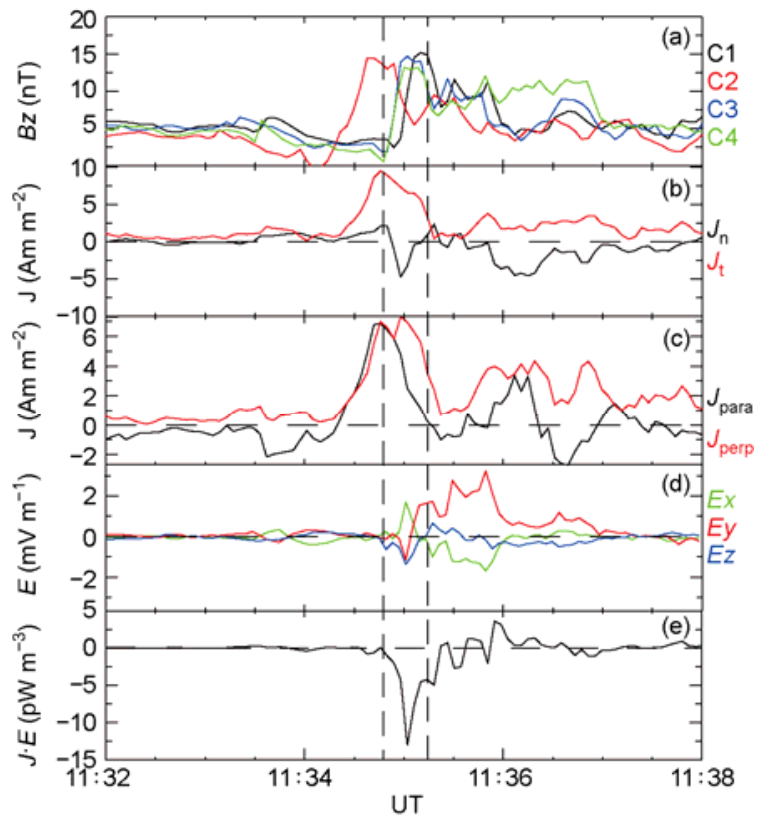

Figure 3 (a) $B z$ variations observed by four Cluster satellites; (b) current components normal and tangential to the DF; (c) field-aligned and perpendicular components of current calculated by curlometer technique; (d) electric field components; and (e) $J \cdot E$, electromechanical energy conversion term in Poynting's equation.

normal direction of the DF. The differences of the direction calculated by two methods are quite small, which is also proved by Nakamura et al. [1], indicating that the DF observed by Cluster is a two dimensional structure, like a thin current sheet [7]. In order to get the current distribution, the magnetic field data of four satellites are used in multi-spacecraft coulometer technique. The divergence of the magnetic field divided by the curl of it is far less than 0.5 , indicating the current calculation result is reliable. As the tangential component to the DF plane $J_{\mathrm{t}}$ is much larger than the normal component $J_{\mathrm{n}}$ (Figure $3(\mathrm{~b})$ ), it is suggested that most of the current is flowing in the DF plane, consistent with the idea that the DF is a thin current sheet. Figure 3(c) provides the parallel and perpendicular components of current density to the magnetic field. The parallel current density, which is about $6 \mathrm{nA} \mathrm{m}^{-2}$ at the leading edge of the DF, agrees well with the $5.9 \mathrm{nA} \mathrm{m}^{-2}$ calculated by differential particle flux of electrons. This result implied that the main field-aligned current at the DF is carried by the electrons around $1 \mathrm{keV}$. 4s-resolution electric field obtained from EFW is shown in Figure 3(d). Positive peak of Ex and negative peak of $E y$ and $E z$ are found within the DF structure. Strong electric field (about $4 \mathrm{mV} \mathrm{m}^{-1}$ ) can be found during the high speed flow. The electromechanical energy conversion term in Poynting's equation $J \cdot E$ is plotted in Figure 2(e). As the DF and BBF moves toward the Earth, the plasma has to slow down, and the DF becomes a "dynamo" with $J \cdot E<0$, indicating the structure will release energy to the ambient region [14].
Figure 4 shows the electron differential energy flux at pitch angle $180^{\circ}$ observed by 4 Cluster satellites. The magnetic field $B z$ component are plotted on top of the energy spectra. The electron injection coincident with the narrow $B z$ decrease preceding the sharp increase, and the duration of the high electron flux and the increase of the magnetic field $B z$ component fit each other surprisingly well. It is quite clear that the enhancements of the anti-field aligned electrons are recorded by all the four satellites, indicating that the structure is very stable, lasting for at least $90 \mathrm{~s}$, and the increases of electron flux are closely associated with the dipolarization front.

The ion spectra obtained by CIS-HIA are displayed in Figure 5(a) and the azimuthal angle distribution of ions is shown in Figure 5(b). The azimuthal angle is defined as the detector samples the particle flux from different directions, with $0^{\circ}$ coming from the tail and $90^{\circ}$ from the dawn ward. We can see that before the arriving of the main body of the high speed flow around $11: 35: 45$, the flow has an apparently deviation from the flow direction. A careful inspection shown that the flow mainly deflected to the dawn ward other than the dusk ward, with the flux of $-90^{\circ}$ stronger than the flux at $90^{\circ}$. It is consistent with the calculated propagation speed of the DF in the $Y$ direction. $\mathrm{C} 1$ stayed at the dawn side of the flow before the BBF arrived, so a strong (compared with quiet background) dawn ward reflection can be observed before the main part of the flow.

\section{Discussion and summary}

On July 22, 2001, four Cluster satellites observed a magnetic structure which has the common character of a DF: a sharp $B z$ jump followed by a high speed flow. A 2D structure of the front was confirmed and based on the propagation speed and the duration of the DF, the scale of DF structure is estimated to be around $600 \mathrm{~km}$, which is just one ion gyro-radius. Bi-directional electron anisotropy at the DF is clearly observed for the first time. The upward streaming electrons are not balanced by the downward flux, which can account for the field aligned current (FAC) density at the DF calculated from the magnetic field data.

Previous studies found that Fermi acceleration of electrons behind the dipolarization fronts may cause a bidirectional distribution of electrons like what is observed in this paper. Fu et al. [8] suggested that Fermi acceleration mechanism works in a decaying field pileup region (FPR) case, leading to the enhancement of electrons fluxes in the parallel and anti-parallel direction. In a growing FPR, the enhancement of electrons appears mainly in the perpendicular direction, suggesting a betatron acceleration mechanism. However, it should be noted that both Fermi and betatron acceleration studied by Fu et al. [8] work for high energy electrons $(>40 \mathrm{keV})$ after the DF structure. Different from their events [8], in the July 22 event, strong increase of 


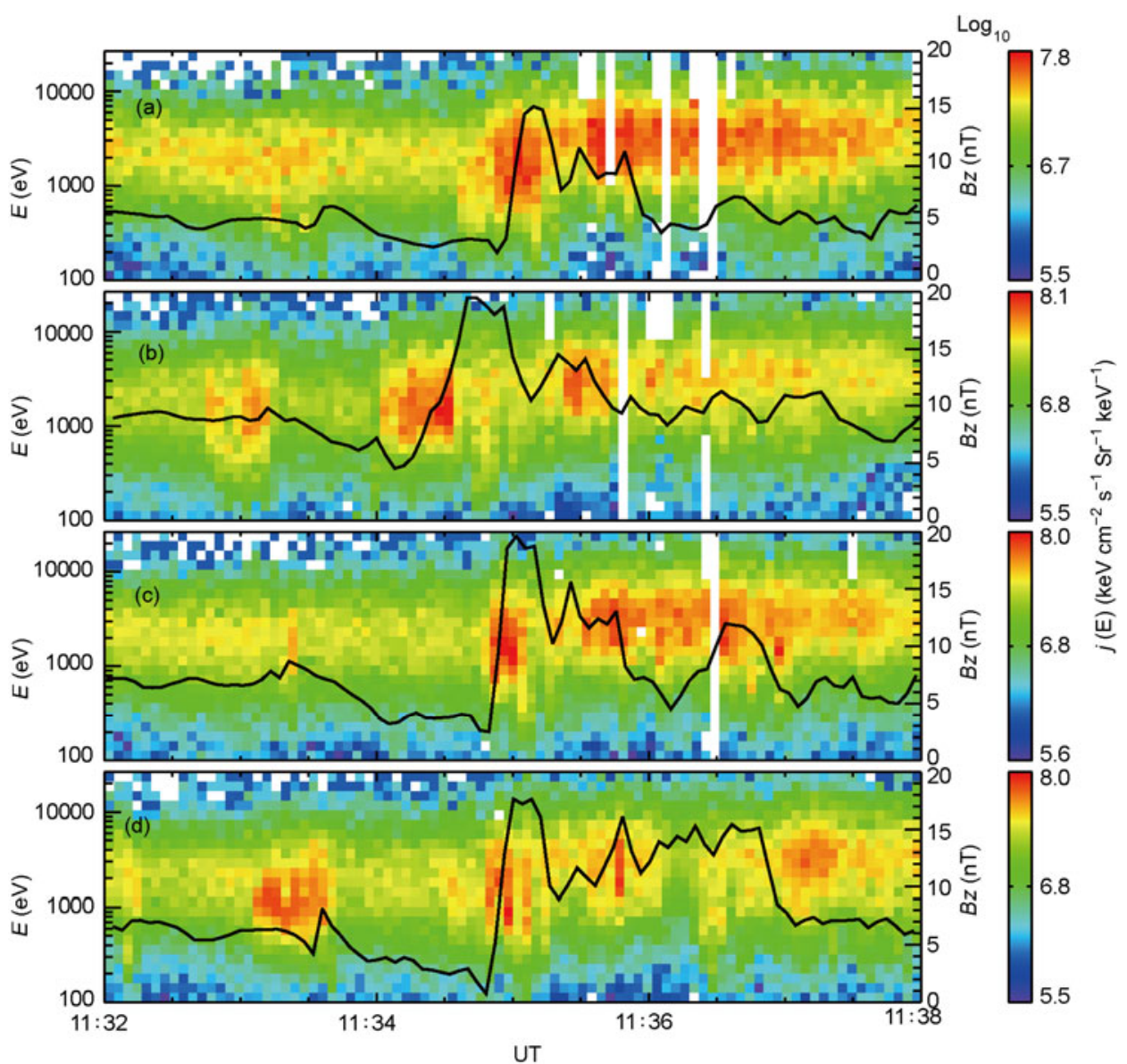

Figure 4 Energy spectra of electrons at pitch angle $180^{\circ}$ observed by the four Cluster satellites, with the magnetic field $B z$ component plotted on top of the spectra ((a)-(d) correspond to $\mathrm{C} 1-\mathrm{C} 4$, respectively).

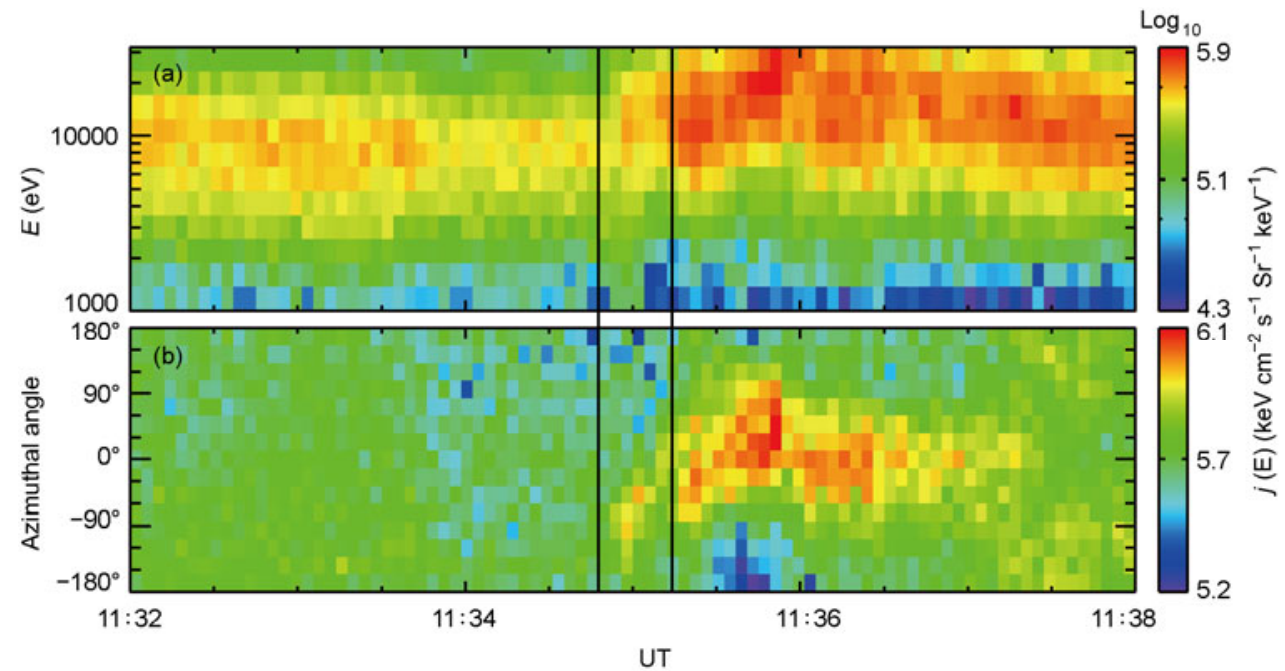

Figure 5 (a) Color coded ion energy spectra of differential energy flux (b) Azimuthal angle distribution of ion differential energy flux (10-30 keV).

electrons at the DF is sitting in the energy range between $200 \mathrm{eV}$ and $2 \mathrm{keV}$, with a peak around $1 \mathrm{keV}$. These electrons could not be produced by the Fermi mechanism and the reasons are the following. Firstly, it is well known that Fermi acceleration works when the second adiabatic invar- iant is conserved. Therefore, it should work for particle of any energy range which can satisfy the adiabatic conditions. However, in this case, the increase flux of bidirectional electrons is confined within a pretty narrow energy range just around $1 \mathrm{keV}$. Secondly, assuming the Fermi mecha- 
nism works in the tail, the radial distance to the Earth can be treated as the length of magnetic field line in the mid tail region. The bounce period of $1 \mathrm{keV}$ electrons is $\sim 4.96 \mathrm{~s}$. In our observation the cigar distribution only lasts for about $8 \mathrm{~s}$ right at the DF, it implied the $1 \mathrm{keV}$ electrons could only have 1-2 bounce cycles during such a time scale. As the propagation speed of DF is about $41 \mathrm{~km} \mathrm{~s}^{-1}$, the radial distance change could be estimated to be $0.05 \mathrm{R}_{\mathrm{E}}$. The length of magnetic field line would not shrink too much in this period, and Fermi effect would not be very strong. The third reason is that Fermi acceleration will not produce asymmetric flux between the $0^{\circ}$ and $180^{\circ}$ pitch angle electrons, which is quite obvious in our case. We show in Figure 2 that the slope of the electron spectra in the energy range of 2-30 keV do not change during the dipolarization, indicating that somehow these electrons might go through a Fermi like process. It is quite clear that those electrons around 1 $\mathrm{keV}$, which forming the field-aligned current for the DF, have totally different behavior compared with the electrons in the energy range of a few tens of $\mathrm{keV}$.

It is well accepted that the downward current electron beams play an important role in ionosphere-magnetosphere coupling. Upward streaming electrons associated with downward field align current at altitudes of $1 \mathrm{R}_{\mathrm{E}}$ over the auroral zone have been observed over the years [15]. However, those electrons, peak at $100-200 \mathrm{eV}$, have a maximum flux at an order of $10^{10} \mathrm{~cm}^{-2} \mathrm{~s}^{-1} \mathrm{Sr}^{-1} \mathrm{keV}^{-1}$. Observations by FAST satellite have demonstrated that downward current electron beam is a very common feature in the auroral zone $[16,17]$. The observed downward current electron beam has a broad spectrum between $100 \mathrm{eV}$ and $5 \mathrm{keV}$, with peak energy near $1 \mathrm{keV}$, which is pretty much similar with what we present in this paper.

It is found that anti-parallel electrons have higher flux than parallel electrons in the DF structure, leading to a field-aligned current. The relation of field-aligned current and the braking or deflection of the high speed flows has been studied [17-20]. Vortex-like plasma flow induced field-aligned current has been observed at the high latitude and $1 \mathrm{keV}$ outflowing oxygen ions from the ionosphere are reported together with the current [21]. Figure 6 gives a schematic plot to illustrate the field-aligned current and the braking of the high speed flow. In our event, the azimuthal deflection of the flow can also be seen right at the DF

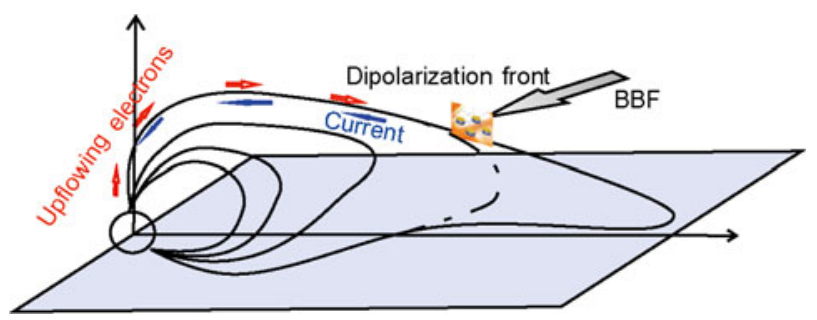

Figure 6 Schematic illustration of the upflowing electrons and the dipolarization in front of a BBF.
(Figure 4(b)), indicating a sign of braking induced flow vortex. The observed field-aligned current is also consistent with the plasma flow pattern.

Cluster STAFF instrument observed only broadband electrostatic noises (not shown here) rather than a structured electric field carried by waves, e.g. solitary waves, suggesting the observed downward current electron beams (peaked at $1 \mathrm{keV}$ ) may refer to the acceleration at the auroral zone. According to T96 model and IGRF model, it is found that the magnetic field of source region of these $\sim 1 \mathrm{keV}$ electrons is almost equal to the magnetic field of $2.7 \mathrm{R}_{\mathrm{E}}$ height at polar region. However, how these electron beams formed is still an open question. There are at least three acceleration mechanisms being proposed to account for the downward current electron beams: (1) stationary, oblique double layer [17,22]; (2) time-dependent upward moving double layers with variable electron energies [23,24]; and (3) a quasisteady acceleration mechanism with U-shaped potential structure [16], with the possible location of the acceleration below the $B / n$ peak ( $B$ and $n$ being the magnetic field and electron number density, respectively) [25].

Nevertheless, the observational facts made in this paper provide new clues to understand the acceleration process of the downward current electron beams. The downward current electron beams are quasi-steady during all four Cluster spacecraft crossing time of $80 \mathrm{~s}$ (Figure 4). Regions of downward current thus require a quasi-steady acceleration mechanism to maintain the current flow and the electron beams. A time-dependent process like upward moving double layers, thus, may be ruled out to interpret the dipolarization front associated electron beams. Further, stationary double layer requires heated ions to maintain the double layer structure [16], however, Cluster all four spacecrafts only observed electron beams, without ion beams, suggesting a stationary double layer existing in the auroral zone is hardly to explain the observations reported in this paper. Thus, we propose that the downward current electron beams observed by Cluster may be accelerated by a quasi-steady acceleration mechanism with U-shaped potential structure as suggested by Carlson et al. [16] and Cran-McGreehin and wright [25]. The dipolarization front together with a high speed plasma flow may help to build a U-shaped potential structure in the auroral zone region.

This work was supported by the National Natural Science Foundation of China (40874086 and 41031065) and the National Basic Research Program of China (2011CB811404). We thank Dr. George Parts for valuable discussions and constructive suggestions. We thank Cluster Active Archive for providing data and we also acknowledge the contributions of all CLUSTER teams to make these data available at the CAA website.

1 Nakamura R, Baumjohann W, Klecker B, et al. Motion of the dipolarization front during a flow burst event observed by Cluster. Geophys Res Lett, 2002, 29: 1942-1945

2 Apatenkov S V, Sergeev V A, Kubyshkina M V, et al. Multi-spacecraft observation of plasma dipolarization/injection in the inner mag- 
netosphere. Ann Geophys, 2007, 25: 801-814

3 Lui A T Y, Angelopoulos V, LeContel O, et al. Determination of the substorm initiation region from a major conjunction interval of THEMIS satellites. J Geophys Res, 2008, 113: A00C04

4 Mende S, Angelopoulos V, Frey H U, et al. Timing and location of substorm onsets from THEMIS satellite and ground based observations. Ann Geophys, 2009, 27: 2813-2830

5 Runov A, Angelopoulos V, Sitnov M I, et al. THEMIS observations of an earthward-propagating dipolarization front. Geophys Res Lett, 2009, 361: L14106

6 Sergeev V, Angelopoulos V, Apatenkov S, et al. Kinetic structure of the sharp injection/dipolarization front in the flow-braking region. Geophys Res Lett, 2009, 362: L21105

7 Zhou M, Ashour-Abdalla M, Deng X, et al. THEMIS observation of multiple dipolarization fronts and associated wave characteristics in the near-Earth magnetotail. Geophys Res Lett, 2009, 362: L20107

8 Fu H S, Khotyaintsev Y V, Andre M, et al. Fermi and betatron acceleration of suprathermal electrons behind dipolarization fronts. Geophys Res Lett, 2011, 38: L16104

9 Escoubet C, Fehringer M, Goldstein M. The Cluster mission. Ann Geophys, 2001, 19: 1197-1200

10 Angelopoulos V, Baumjohann W, Kennel C F, et al. Bursty bulk flows in the inner central plasma sheet. J Geophys Res, 1992, 97: 4027-4039

11 Baumjohann W, Paschmann G, Luehr H. Characteristics of high-speed ion flows in the plasma sheet. J Geophys Res, 1990, 95: 3801-3809

$12 \mathrm{Lu} \mathrm{S}, \mathrm{Lu} \mathrm{Q} \mathrm{M}$, Cao Y, et al. The effects of the guide field on the structures of electron density depletions in collisionless magnetic reconnection. Chin Sci Bull, 2011, 56: 48-52

13 Chen C X, Wolf R A. Interpretation of high-speed flows in the plasma sheet. J Geophys Res, 1993, 98: 21409-21419

14 Zong Q G, Fritz T A, Pu Z Y, et al. Earthward flowing plasmoid: Structure and its related ionospheric signature. J Geophys Res, 2007,
112: A07203

15 Sharp R D, Shelley E G, Johnson R G, et al. Counterstreaming electron beams at altitudes of about $1 \mathrm{RE}$ over the auroral zone. J Geophys Res, 1980, 85: 92-100

16 Carlson C W McFadden J P, Ergun R E, et al. FAST observations in the downward auroral current region: Energetic upgoing electron beams, parallel potential drops, and ion heating. Geophys Res Lett, 1998, 25: 2017-2020

17 Ergun R E, Andersson L, Main D, et al. Auroral particle acceleration by strong double layers: The upward current region. J Geophys Res, 2004, 109: A12220

18 Keiling A, Angelopoulos V, Runov A, et al. Substorm current wedge driven by plasma flow vortices: THEMIS observations. J Geophys Res, 2009, 114: A00C22

19 Hasegawa A. Generation of field aligned current during substorm. In: Akasofu S I, ed. Dynamics of the Magnetosphere. Dordrecht: D. Reidel, 1998. 529-542

20 Fu S Y, Shi Q Q, Wang C, et al. High-speed flowing plasmas in the Earth's plasma sheet. Chin Sci Bull, 2011, 56: 1-6

21 Zong Q G, Wang Y F, Yang B, et al. Vortex-like plasma flow structures observed by Cluster at the boundary of the outer radiation belt and ring current: A link between the inner and outer magnetosphere. J Geophys Res, 2009, 114: A10211

22 Block L P. Potential double layers in the ionosphere. Cosmic Electrodyn, 1972, 3: 349-376

23 Andersson L, Ergun R E. Acceleration of antiearthward electron fluxes in the auroral region. J Geophys Res, 2006, 111: A07203

24 Andersson L, Ergun R E, Newman D L, et al. Characteristics of parallel electric fields in the downward current region of the aurora. Phys Plasmas, 2002, 9: 3600-3609

25 Cran-McGreehin A P, Wright A N. Current-voltage relationship in downward field-aligned current region. J Geophys Res, 2005, 110: A10S10

Open Access This article is distributed under the terms of the Creative Commons Attribution License which permits any use, distribution, and reproduction in any medium, provided the original author(s) and source are credited. 